\begin{abstract}
This paper substantiates the pulse method for determining the time parameter for fire detectors with a thermoresistive sensing element - the time constant. The method is based on using the JouleLenz effect, which manifests itself when an electric current pulse passes through the thermoresistive sensing element of fire detectors. Thermal processes in such a sensing element are described by a mathematical model that belongs to the class of equations of mathematical physics. The solution to the differential equation of this class was derived using the Hankel integral transformation and is represented as a series relative to the Bessel functions. The resulting solution is used to construct a mathematical model of a thermoresistive sensing element in the form of a transfer function, which takes the form of the transfer function of the inertial link. To trigger the thermoresistive sensing element of fire detectors, a single pulse of electric current in the shape of a rectangular triangle is used. The integral Laplace transformation was applied to mathematically describe the response of a thermoresistive sensing element to the thermal effect of such a test influence. To obtain information about the time parameter of fire detectors with a thermoresistive sensing element, the ratio of its output signals is used, which are measured in the a priori defined moments. A two-parametric expression was built to determine the time parameter of fire detectors; a verbal interpretation of the pulse method to determine it was provided. The implementation of this method ensures the invariance of the time parameter of fire detectors with a thermoresistive sensing element relative to the amplitude of a single pulse of an electric current, as well as relative to the parameter that is included in its transfer coefficient

Keywords: fire detector, thermoresistive sensing element, Joule-Lenz effect, time parameter
\end{abstract}

\section{SUBSTANTIATING THE PULSE METHOD FOR DETERMINING THE TIME PARAMETER OF FIRE DETECTORS WITH A THERMORESISTIVE SENSING ELEMENT}

\author{
Yaroslav Kozak \\ Adjunct
}

Department of Fire Tactics and Emergency Rescue Operations

Lviv State University of Life Safety Kleparivska str., 35, Lviv, Ukraine, 79007

Yuriy Abramov

Doctor of Technical Sciences, Professor, Chief Researcher

Research Center*

Oleks i B a s manov Corresponding author

Doctor of Technical Sciences, Professor, Chief Researcher

Scientific Department on Problems of Civil Defense, Technogenic and Ecological Safety of the Research Center*

E-mail: oleksii.basmanov@nuczu.edu.ua *National University of Civil Defence of Ukraine Chernyshevska str., 94, Kharkiv, Ukraine, 61023
Received date 19.10.2021

Accepted date 29.11.2021 Published date 21.12.2021
How to Cite: Kozak, Y., Abramov, Y., Basmanov, O. (2021). Substantiating the pulse method for determining the time parameter of fire detectors with a thermoresistive sensing element. Eastern-European Journal of Enterprise Technologies, 6 (5 (114)), 49-55. doi: https://doi.org/10.15587/1729-4061.2021.244235

\section{Introduction}

One of the radical ways to reduce fire damage is to identify dangerous fire factors at the initial stage. The greatest effect in the detection of such factors can be obtained only with the help of automatic systems. The effectiveness of such systems is primarily determined by the degree of perfection of the technical characteristics of the sensors of primary information - fire detectors, as well as the efficiency of their operating system [1]. One of the types of fire detectors is the thermal fire detector. The system of operation of such detectors involves the implementation of two classes of their tests - stationary, or autonomous tests, and operational, or object tests. The peculiarity of the first class of tests is that they do not apply to fire detectors that are located at protected facilities. For the second class of tests, it is characteristic that their implementation does not determine the assessment of their parameters and characteristics. The peculiarity of both classes of testing of thermal fire detec- tors is that they do not provide for determining such a time parameter as a time constant. It should be noted that the European standard EN-54 regulates the value of a time constant for thermal fire detectors. These circumstances predetermine the need to devise theoretical bases for object tests of thermal fire detectors, which would ensure at their implementation obtaining the estimates for the basic characteristics of detectors.

Thus, it is a relevant task to devise theoretical foundations using opportunities arising from the principles of building thermal fire detectors. From this point of view, of interest is to apply the Joule-Lentz effect relative to fire detectors with a thermoresistive sensing element.

\section{Literature review and problem statement}

Among the physical concepts employed to build thermal fire detectors, the thermoresistive effect occupies one of the 
leading positions. This is due to the simplicity of implementation and high sensitivity [2] of such devices.

The perfection of the technical characteristics of fire detectors depends on the quality of mathematical support at all stages of their life cycle. Paper [3] reports the results from a study at the Polish Air Force Technology Institute (AFIT) on the simulation of dynamic properties of fire detectors used in the aircraft fire extinguishing system. However, the authors disregarded resolving the issue related to identifying the parameters of fire detectors when they work under different modes. In particular, the possibility to change the triggering time of fire detectors is noted in [4]. To mitigate this effect, the authors propose increasing the number of fire detectors but there is no justification for the number of such fire detectors. In addition, study [5] notes that this approach is not effective and leads to an increase in financial costs. In [6], attention is paid to that the time of fire detector triggering is determined both by the nature and parameters of the input impact and by the properties of the fire detector. It should be noted that the dynamic properties of thermal fire detectors are fully determined by such a time parameter as a time constant. In most works, the first component of the triggering time is considered. This approach was used in [7]; the authors proposed a mathematical model to describe the dynamics of ambient temperature in the initial stage of fire. They solved the differential equation of heat transfer from the site to the sensor under typical temperature conditions. The study results were used to calculate the triggering time of several irrigation systems for fire extinguishing. However, at the same time, the impact of the dynamic properties of sensors at the time of triggering such systems was not considered. In [8], numerical simulation was carried out of the fire in the cargo compartment of the DC-10 (USA) aircraft at different ventilation intensities. The research is aimed at identifying the impact of fire conditions parameters on the activation of fire detectors. However, the dynamic properties of fire detectors were not clearly considered in those studies. In [9], the authors note that the heat flow from the internal combustion engine affects the fire detector triggering during the fire detector operation. The algorithm of operation of thermal fire detectors was proposed, which is based on measuring the minimum static temperature of the detector actuation, as well as the value of the temperature increase rate. That approach was verified in [10]. It should be noted that the general disadvantage of the cited works is that they do not consider the component of the triggering time of fire detectors, which is due to their dynamic properties. This applies to such a parameter as the time constant of detectors. A similar approach is characteristic of [11], which examines the intelligent fire detection system at its initial stage. Such a system was implemented in the botanical park Faruk Yalchin in Darija (Turkey). The algorithm of the system includes real-time fire analysis (fire spread rate, propagation direction) but the properties of detectors were not taken into account. That also applies to [12], in which, to obtain a predictive estimate of the time of fire detector triggering, a fire model was built for its stable and quasi-stationary phase. To obtain an estimate of the triggering time, it was assumed that its time parameter did not differ within the tolerance from the rated value. In [13], it is noted that the time of fire detectors triggering, in addition to the parameters and characteristics of the fire, depends on the distance to it. A series of tests using different fire sizes at different vent speeds and ignition locations was conducted at the NIOSH Safety Research Coal Mine in Bruceton, Pennsylvania [14]. As a result of those tests, assessments of the time of fire hazardous factors were obtained. As a result, the locations of such sensors were determined. It is concluded that according to the results of those studies, appropriate fire sensors can be selected. However, it should be noted that such a conclusion can only be extended to the type of sensors, and not to their parameters and characteristics. In particular, this applies to their time parameters. In a series of works on the issue of fire detectors, attention is drawn to their false triggering. Paper [15] argues that there were more than 300,000 false triggerings recorded in the UK in two years. Such false triggering is the result of improper installation, negligent maintenance, as well as environmental impact, and anthropogenic factors. One option to reduce the number of cases of false triggering is to use sensors instead of detectors [16] in conjunction with the adaptive method of processing measurement results in a multi-layered perceptron-based system. The disadvantage of that approach is that it is not supposed to diagnose sensors of primary information. Another option to reduce false triggering is to use neural networks [17], or fuzzy logic [18]. In the first case, the reduction of false triggering is provided with the software by increasing the duration of training. In the second case, fire detection is carried out by forecasting using fuzzy logic. It should be noted that the disadvantage of such options is that they do not take into account the technical condition of the sensors of primary information. A promising option is a variant that provides for determining the technical condition of fire detectors. To this end, it is advisable to conduct their tests, the result of which should be to obtain assessments of their main characteristics [19]. Paper [19], as well as study [20], report the results of tests of fire detectors, during which estimates for one of their time parameters were obtained - the triggering time. In [21], the results of obtaining estimates of the actuation time and the actuation time index (RTI) for thermal fire detectors of the maximum type are given. All those tests have a common drawback - when they are carried out, such a time parameter of the fire detector as its time constant is not determined. In addition, those tests are laboratory-type tests; their implementation within the system of operation of fire detectors is problematic. This is especially true of testing fire detectors directly at protected sites. The review of methods and means for testing thermal fire detectors, which was carried out in [22], indicates the following:

- when conducting stationary or autonomous tests, the amount of time of fire detector triggering (using thermal cameras) is determined while the time parameter is not defined - a time constant (although this parameter is regulated by the European standard EN-54);

- when conducting operational or object tests, in most cases, the time parameters of fire detectors are not determined. The result of such tests, for example, according to the standard APSAD R7 (France) or the standard BS 5839 1:2002 Clause 45.4 (Great Britain) is the fact of triggering the detector.

The data above indicate the issue related to improving the efficiency of the system of operation of thermal fire detectors by advancing the methods and means for their testing. One of the areas to address this issue is to use potential opportunities, which are laid down in the principle of construction of fire detectors. In particular, this applies to fire detectors whose principle of operation is based on the thermoresistive effect. As regards this type of fire detectors, thermal impact on their sensing element during tests can be exerted by using the Joule-Lentz effect. This opens up opportunities for testing fire detectors of this type directly at protected areas. 


\section{The aim and objectives of the study}

The purpose of this work is to substantiate the method for determining the time parameter of fire detectors with a thermoresistive sensing element, based on the use of the Joule-Lenz effect in the formation of test exposure. This would make it possible to conduct tests of fire detectors of this type directly at protected sites, completely under an automatic mode.

To accomplish the aim, the following tasks have been set:

- to describe mathematically those thermal processes that take place in the thermoresistive sensing element of a fire detector in the presence of an internal heat source due to the manifestation of the Joule-Lenz effect;

- to substantiate the structure and parameters for the model of a thermoresistive sensing element of fire detectors in the class of transfer functions;

- to formalize with the help of the operator mathematical models of a thermoresistive sensing element in the fire detector the procedure for implementing a method of determining its time parameter.

\section{The study materials and methods}

When justifying the method for determining the time parameter of fire detectors with a thermoresistive sensing element, we use mathematical models in the class of equations of mathematical physics, and in the operator form. To solve the equation of mathematical physics, the integral Hankel transformation is applied. When constructing a model of the thermoresistive sensing element of a fire detector in the operator form, as well as for a mathematical description of its response to the thermal action of an electric current, we employ the integral Laplace transformation.

\section{Results of research into substantiating the method of determining the time parameter of fire detectors}

\section{1. The mathematical description of thermal process-} es in the thermoresistive sensing element of a fire detector

When an electric current $i(t)$ passes through the thermoresistive sensing element of a fire detector, a thermal effect would occur, in accordance with the Joule-Lenz law. As a result, the temperature of the thermoresistive sensing element would change. The description of thermal processes in such a sensing element of the fire detector can be represented as a differential equation:

$$
\frac{\partial \theta(r, t)}{\partial t}=a\left[\frac{\partial^{2} \theta(r, t)}{\partial r^{2}}+\frac{1}{r} \frac{\partial \theta(r, t)}{\partial r}\right]+\phi(r, t),
$$

with the initial and boundary conditions

$$
\theta(r, 0)=0 ; \quad \frac{\partial \theta(0, t)}{\partial t}=0 ; \quad \frac{\partial \theta(R, t)}{\partial r}=-h \theta(R, t),
$$

where $a$ is the coefficient of thermal conductivity of the thermoresistive sensing element; $h$ is the relative heat exchange coefficient; $R$ is the radius of the thermoresistive sensing element; $\varphi(\mathrm{t})$ is a function that describes the internal heat source, caused by the thermal effect of electric current $i(t)$

$$
\phi(r, t)=K i^{2}(t)
$$

$K=$ const is a parameter.

In expressions (1) and (2), we consider

$\theta(r, t)=T(r, t)-T_{0}$,

where $T(r, t), T_{0}$ is the temperature of the thermoresistive element and its initial temperature, respectively.

If one applies the Hankel integral transformation to differential equation (1), it will be transformed as follows

$$
\frac{d \bar{\theta}\left(\frac{\mu_{n}}{R}, t\right)}{d t}=a H\left[\frac{\partial^{2} \theta(r, t)}{\partial r^{2}}+\frac{1}{r} \frac{\partial \theta(r, t)}{\partial r}\right]+\bar{\phi}\left(\frac{\mu_{n}}{R}, t\right),
$$

where

$$
\begin{aligned}
& \bar{\theta}\left(\frac{\mu_{n}}{R}, t\right)=H[\theta(r, t)]=\int_{0}^{R} r J_{0}\left(\frac{\mu_{n} r}{R}\right) \theta(r, t) \mathrm{d} r ; \\
& \bar{\phi}\left(\frac{\mu_{n}}{R}, t\right)=H[\phi(r, t)]=\int_{0}^{R} r J_{0}\left(\frac{\mu_{n} r}{R}\right) \phi(r, t) \mathrm{d} r ;
\end{aligned}
$$

$\mu_{n}$ is the $n$-th root of the transcendent equation

$$
h R J_{0}(\mu)-\mu J_{1}(\mu)=0 ;
$$

$J_{0}(\mu), J_{1}(\mu)$ are the Bessel functions of the first kind of zero and first orders, respectively; $H$ is the Hankel cumulative transformation operator.

After two-time integration for parts of the additive component in (5), taking into account the differential Bessel equation with respect to the function $J_{0}\left(\mu_{n} r / R\right)$, as well as boundary conditions (2), the differential equation (5) will be transformed to the form

$$
\frac{d \bar{\theta}\left(\frac{\mu_{n}}{R}, t\right)}{d t}+a\left(\frac{\mu_{n}}{R}\right)^{2} \bar{\theta}\left(\frac{\mu_{n}}{R}, t\right)=\frac{K h R^{3} J_{0}\left(\mu_{n}\right)}{\mu_{n}^{2}} i^{2}(t) .
$$

The solution to this differential equation is

$$
\begin{aligned}
& \bar{\theta}\left(\frac{\mu_{n}}{R}, t\right)=\frac{K h R^{3} J_{0}\left(\mu_{n}\right)}{\mu_{n}^{2}} \times \\
& \times \int_{0}^{t} i^{2}(\varepsilon) \exp \left[-a\left(\frac{\mu_{n}}{R}\right)^{2}(t-\varepsilon)\right] \mathrm{d} \varepsilon .
\end{aligned}
$$

This solution is related to the solution $\theta(r, t)$ to the differential equation (1) via the reverse Hankel transformation $H^{-1}$ (reverse formula)

$$
\begin{aligned}
& \theta(r, t)=H^{-1}\left[\bar{\theta}\left(\frac{\mu_{n}}{R}, t\right)\right]= \\
& =\frac{2}{R^{2}} \sum_{n=1}^{\infty} \frac{\mu_{n}^{2} J_{0}\left(\frac{\mu_{n} r}{R}\right) \bar{\theta}\left(\frac{\mu_{n}}{R}, t\right)}{J_{0}^{2}\left(\mu_{n}\right)\left[(h R)^{2}+\mu_{n}^{2}\right]}= \\
& =2 k h R \sum_{n=1}^{\infty} \frac{J_{0}\left(\frac{\mu_{n} r}{R}\right)}{J_{0}\left(\mu_{n}\right)\left[(h R)^{2}+\mu_{n}^{2}\right]} \times \\
& \times \int_{0}^{t} i^{2}(\varepsilon) \exp \left[-a\left(\frac{\mu_{n}}{R}\right)^{2}(t-\varepsilon)\right] \mathrm{d} \varepsilon .
\end{aligned}
$$


Due to the small size of the thermoresistive sensing element of a fire detector, it is advisable to use the temperature averaged across its volume, that is,

$$
\begin{aligned}
& \theta(t)=\frac{2}{R^{2}} \int_{0}^{R} r \theta(r, t) \mathrm{d} r= \\
& =4 K(h R)^{2} \sum_{n=1}^{\infty}\left[\mu_{n}^{2}\left[(h R)^{2}+\mu_{n}\right]\right]^{-1} \times \\
& \times \int_{0}^{t} i^{2}(\varepsilon) \exp \left[-a\left(\frac{\mu_{n}}{R}\right)^{2}(t-\varepsilon)\right] \mathrm{d} \varepsilon .
\end{aligned}
$$

This expression is general for determining the reaction of the thermoresistive sensing element to the thermal action of the electric current flowing through it.

\section{2. The structure and parameters of the model of a thermoresistive sensing element in the class of transfer} functions

Bearing in mind that the fire detector is a dynamic element of the fire hazard detection system, it is advisable to represent a model of the thermoresistive sensing element in the form of a transfer function

$$
W(p)=L[\theta(t)]\left[L\left[i^{2}(t)\right]\right]^{-1},
$$

where $L$ is the Laplace cumulative transformation operator; $p$ is a complex variable.

To determine the transfer function (13), we use expression (12) provided

$$
i(t)=I \cdot 1(t)
$$

where $I=$ const, $1(t)$ is the Heaviside function.

Expression (12), considering (14), takes the form

$$
\begin{aligned}
& \theta(t)=4 K I^{2}(h R)^{2} R^{2} a^{-1} \times \\
& \times \sum_{n=1}^{\infty}\left[\mu_{n}^{4}\left[(h R)^{2}+\mu_{n}^{2}\right]\right]^{-1}\left[1-\exp \left[-a\left(\frac{\mu_{n}}{R}\right)^{2} t\right]\right] .
\end{aligned}
$$

It follows from expressions (14) and (15)

$$
\begin{aligned}
& L[\theta(t)]=4 K I^{2}(h R)^{2} R^{2}(a p)^{-1} \times \\
& \times \sum_{n=1}^{\infty}\left[\mu_{n}^{4}\left[(h R)^{2}+\mu_{n}^{2}\right]\left[\left(R \mu_{n}^{-1}\right)^{2} a^{-1} p+1\right]\right]^{-1}, \\
& L\left[i^{2}(t)\right]=I^{2} p^{-1},
\end{aligned}
$$

as a result, the following expression holds for the transfer function $W(p)$

$$
W(p)=4 K \sum_{n=1}^{\infty} A_{n} \tau_{n}\left(\tau_{n} p+1\right)^{-1}
$$

where $A_{n}$ is the $n$-th reduced transfer factor of the thermoresistive sensing element

$$
A_{n}=(B i)^{2}\left[\mu_{n}^{2}\left[(B i)^{2}+\mu_{n}^{2}\right]\right]^{-1}
$$

$\tau_{n}$ is the $n$-th time constant of the thermoresistive sensing element

$$
\tau_{n}=\left(R \mu_{n}^{-1}\right)^{2} a^{-1}
$$

$B i=h R$ is the Biot number.

Table 1 gives the values of roots $\mu_{n}$ for $B i \leq 1.0$ at $n=1 . .3$.

Table 1

Values for roots $\mu_{n}$

\begin{tabular}{|c|c|c|c|}
\hline$B i$ & $\mu_{1}$ & $\mu_{2}$ & $\mu_{3}$ \\
\hline 0 & 0.0000 & 3.8317 & 7.0156 \\
\hline 0.1 & 0.4417 & 3.8577 & 7.0298 \\
\hline 0.2 & 0.6170 & 3.8835 & 7.0440 \\
\hline 0.3 & 0.7465 & 3.9091 & 7.0582 \\
\hline 0.4 & 0.8516 & 3.9344 & 7.0723 \\
\hline 0.5 & 0.9408 & 3.9594 & 7.0864 \\
\hline 0.6 & 1.0184 & 3.9841 & 7.1004 \\
\hline 0.7 & 1.0893 & 4.0085 & 7.1143 \\
\hline 0.8 & 1.1490 & 4.0325 & 7.1282 \\
\hline 0.9 & 1.2048 & 4.0562 & 7.1421 \\
\hline 1.0 & 1.2558 & 4.0795 & 7.1558 \\
\hline
\end{tabular}

Data in Table 1 indicate that line (19) quickly converges. For example, at $B i=1.0$, the value of coefficient $A_{2}$ is $1.2 \%$ of the value of coefficient $A_{1}$. As a result, with an error not exceeding $1.2 \%$, the expression for (18) takes the following form

$$
W(p)=4 K A \tau(\tau p+1)^{-1}
$$

where $\tau$ is the time constant whose time parameter is described as

$$
\tau=\tau_{1}=\left(R \mu_{1}^{-1}\right)^{2} a^{-1}
$$

$A$ is the reduced transfer coefficient that is described as follows:

$$
A=A_{1}=(B i)^{2}\left[\mu_{1}^{2}\left[(B i)^{2}+\mu_{1}^{2}\right]\right]^{-1} .
$$

For root $\mu_{1}$, according to Table 1, one can write

$$
\begin{aligned}
& \mu_{1}=2094.356 B i^{9}-10615.079 B i^{8}+23082.011 B i^{7}- \\
& -28124.999 B i^{6}-21037.731 B i^{5}-9946.458 B i^{4}+ \\
& +2947.796 B i^{3}-524.212 B i^{2}+ \\
& +51.745 B i-1.630 .
\end{aligned}
$$

Merging (23) and (24) allows us to conclude that at the interval of $0.2 \leq B i \leq 1.0$ the value of $4 A$ with an error not exceeding $4.6 \%$ can be taken equal to unity. Fig. 1 shows a plot of the dependence of error module $\delta=4 A-1.0$ on the Bi number.

As a result, with an error value not exceeding $4.6 \%$, the expression for the transfer function of the thermoresistive sensing element of a fire detector takes the following form

$$
W(p)=K \tau(\tau p+1)^{-1} .
$$

The $\tau$ time parameter fully determines the dynamic properties of a fire detector. As a result, hereafter, the term to be used is the time parameter for a fire detector with a thermoresistive sensing element. 


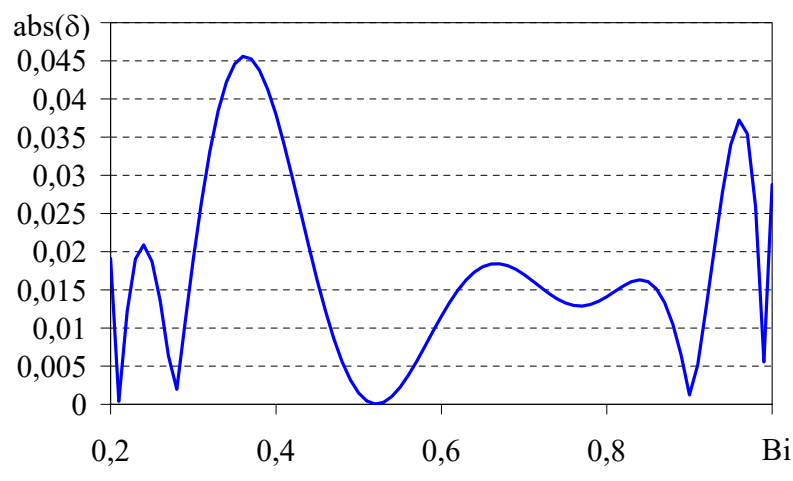

Fig. 1. Dependence of the module of error of divergence on number $\mathrm{Bi}$

5. 3. Formalizing the procedures for implementing the method for determining the time parameter of fire detectors

To trigger a fire detector under its testing mode, a test signal is used in the form of an electric current pulse

$$
i(t)=I\left(1-t t_{0}^{-1}\right)\left[1(t)-1\left(t-t_{0}\right)\right]
$$

where $I, t_{0}$ is the amplitude and pulse duration, respectively.

The reaction of the fire detector to the thermal effect of electric current in the form of (26) is described by the following expression

$$
\begin{aligned}
& \theta(t)=K \tau L^{-1}\left[(\tau p+1)^{-1} L\left[i^{2}(t)\right]\right]=
\end{aligned}
$$

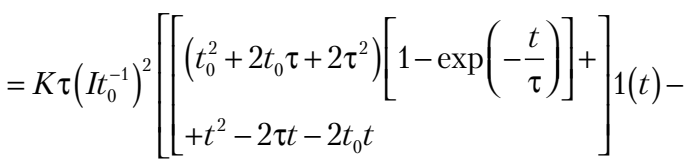

$$
\begin{aligned}
& \left.-\left[\begin{array}{l}
\left(t-t_{0}\right)^{2}-2 \tau\left(t-t_{0}\right)+ \\
+2 \tau^{2}\left[1-\exp \left(-\frac{t-t_{0}}{\tau}\right)\right]
\end{array}\right] 1\left(t-t_{0}\right)\right]
\end{aligned}
$$

where $L^{-1}$ is the Laplace reverse transform operator.

Fig. 2 shows an example of graphical dependences $i(t) I^{-1}$, $\left[i(t) I^{-1}\right]^{2}$ and $m \theta(t)\left[K \tau\left(I t_{0}^{-1}\right)^{2}\right]^{-1}$, where $m$ is scale.

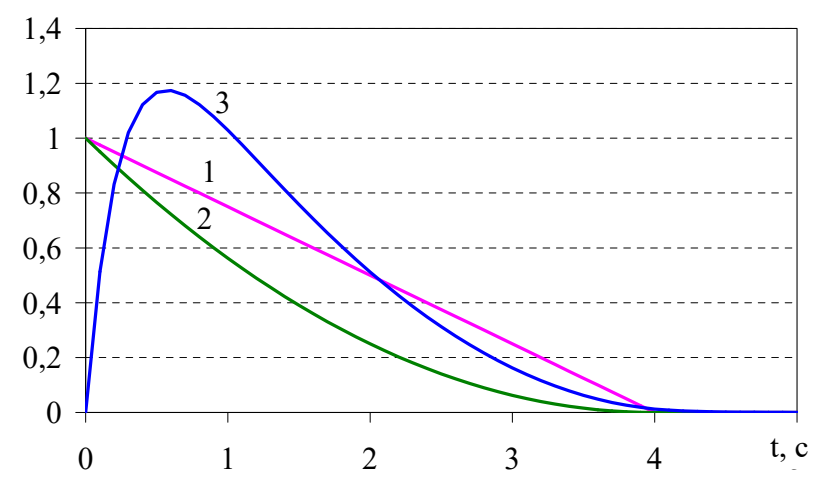

Fig. 2. Dependences: $1-i(t) \digamma^{1} ; 2-\left[i(t) \digamma^{1}\right]^{2}$;

$$
3-m \theta(t)\left[K \tau\left(I t_{0}^{-1}\right)^{2}\right]^{-1} \text { at } m=0.25 ; t_{0}=4.0 \mathrm{~s} ; \tau=0.5 \mathrm{~s}
$$

For time moments $t_{1}, t_{2}$, provided $t \geq 3 \tau$, the following ratio can be written

$$
\begin{aligned}
& \theta\left(t_{1}\right)\left[\theta\left(t_{2}\right)\right]^{-1}=\left[2 \tau^{2}-2\left(t_{1}-t_{2}\right) \tau-\left(t_{1}-t_{0}\right)^{2}\right] \times \\
& \times\left[2 \tau^{2}-2\left(t_{2}-t_{0}\right) \tau-\left(t_{2}-t_{0}\right)^{2}\right]^{-1}=\alpha,
\end{aligned}
$$

hence, the following algebraic equation relative to the time parameter $\tau$

$$
\begin{aligned}
& 2(\alpha-1) \tau^{2}-2\left[\alpha\left(t_{2}-t_{0}\right)-\left(t_{1}-t_{0}\right)\right] \tau+ \\
& +\alpha\left(t_{2}-t_{0}\right)^{2}-\left(t_{1}-t_{0}\right)^{2}=0 .
\end{aligned}
$$

If one accepts $t_{1}=0.5 t_{0}$ and $t_{2}=0.75 t_{0}$ for the ease of technical implementation, the expression to determine the time parameter of a fire detector takes the form:

$$
\tau t_{0}^{-1}=[8(\alpha-1)]^{-1}\left[2-\alpha+\left(6 \alpha-\alpha^{2}-4\right)^{0,5}\right] .
$$

Fig. 3 shows a graphical interpretation of this dependence.

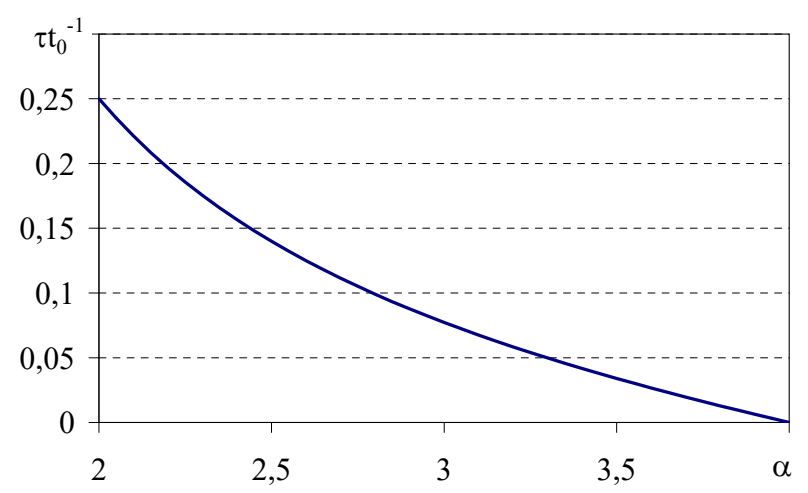

Fig. 3. Dependence $\tau t_{0}^{-1}=f(x)$

It follows from dependence (30) that it is indifferent relative to the amplitude $I$ of the pulse of electric current, as well as the parameter $K$, which depends on the physical parameters of the thermoresistive sensing element of a fire detector. In addition, this dependence is determined only by two parameters (of the possible four) - the duration of the electric current pulse, $t_{0}$, and the ratio of the value of the reaction of a fire detector $\alpha$ at time points $t_{1}$ and $t_{2}$.

A verbal interpretation of the method for determining the time parameters of a fire detector with a thermoresistive sensing element is reduced to a sequence of the following procedures:

- a pulse of the electric current is passed through the thermoresistive sensing element of a fire detector, the shape of which is described by expression (26) and whose parameters are $I$ and $t_{0}$;

- at time points $t_{1}, t_{2}$, one measures a value of the reaction of the thermoresistive sensing element of a fire detector $\theta\left(t_{1}\right)$ and $\theta\left(t_{2}\right)$ to the test signal (26) and determines their ratio $\alpha$;

- using expression (29) or the graphical dependence, which is shown in Fig. 3 , based on the values $\alpha$ and $t_{0}$, one determines a value of the time parameter $\tau$ for a fire detector.

\section{Discussion of results of substantiating the method for} determining the time parameter of fire detectors

Our results regarding the substantiation of the pulse method for determining the time parameter of fire detectors 
with a thermoresistive sensing element are based on the construction of a mathematical description of thermal processes in such a sensing element and their application in the form of the ratio of its output signals in two the a priori predefined time moments.

The peculiarity of the pulse method for determining the time parameter of fire detectors with a thermoresistive sensing element is that when it is implemented, there is no need to use external heat sources.

Underlying the mathematical notation of thermal processes in the thermoresistive sensing element of a fire detector is the use of a heterogeneous equation of non-stable thermal conductivity (1). The heterogeneity of the equation is due to the consideration of the internal heat source, which is a manifestation of the Joule-Lentz effect due to the passage of electric current through the thermoresistive sensing element of the fire detector. To solve such a differential equation, the Hankel integral transformation is used, which provides, unlike the Fourier method, the rapid convergence of the resulting solution in the form of a series. The solution to the equation of non-stationary thermal conductivity is derived in the general form and without restrictions on the function that describes the electric current. This opens up opportunities for the transition to another class of mathematical models of the thermoresistive sensing element of a fire detector. Because the fire detector is a dynamic element of the fire hazard detection system, it is advisable to determine the model of its sensing element in the class of transfer functions. To build such a model, the integral Laplace transformation is employed. The model is a transfer function of the inertial link in the form of (25). The transfer factor of such a link includes two multiplicative components, one of which is the time parameter of a fire detector. This approach to building a model of the thermoresistive sensing element of a fire detector is characterized by an error whose value does not exceed $4.6 \%$ for the value of the Biot number less than unity. The use of this class of models for the thermoresistive sensing element of a fire detector makes it possible to extend the methods of the classical theory of automatic control to solve the problem regarding fire detection systems. In addition, the availability of such mathematical models opens up opportunities to use Drag-and-Drop technologies in the study of both fire detection systems and their elements.

A fire detector is triggered, when determining its time parameter, by the pulse of the electric current of the triangular shape of amplitude $I$ and duration $t_{0}(26)$. The use of the integral Laplace transformation makes it possible to represent the reaction of the thermoresistive sensing element of a fire detector to the test signal (26) in the form (27). This response is described by two additive components in the form of Heaviside functions whose parameters include the time parameter of a fire detector. Expression (27) is used to obtain the ratio of the reaction of the thermoresistive element of a fire detector to the test signal (26) for two points in time. The peculiarity of this ratio is that it is indifferent relative to the amplitude I of the electric current pulse, as well as the parameter $K$, which characterizes the internal heat source (3). Ratio (28) is four-parametric; a second-order algebraic equation (29) follows from it to determine the fire detector's time parameter. Establishing a functional connection between the moments of time in which the ratio is determined (28) and the duration $t_{0}$ of the electric current pulse provides a minimum number of parameters in the expression to determine the time parameter of a fire detector.
The method for determining the time parameter of a fire detector with a thermoresistive sensing element is reduced to the following:

- passing the electric current pulse of form (26) through a sensing element;

- measurement of signals $\theta\left(t_{1}\right)$ and $\theta\left(t_{2}\right)$ at time moments $t_{1}$ and $t_{2}$;

- determining the parameter $\alpha$ according to expression (28);

- using expression (30), or the dependence shown in Fig. 3.

The implementation of this method for determining the time parameter of a fire detector makes it possible to ensure:

- complete automation of this process directly at protected facilities;

- increasing the reliability of the result of controlling thermal fire detectors;

- obtaining forecast estimates regarding both this parameter and the reliability indicators of the fire detector.

The limitations of the pulse method for determining the time parameter of fire detectors with a thermoresistive sensing element include the dependence on the shape of the electric current pulse. However, the general approach to determining the time parameter does not change.

It should be noted that the results of determining the time parameter for thermal fire detectors with a thermoresistive sensing element depend on the temperature change at the interval of measuring its output signals.

Further advancement of this method must involve a study to assess the effect of changes in an ambient temperature between the time points at which the initial signals of the sensing element of a fire detector are measured.

\section{Conclusions}

1. The thermal processes have been mathematically described that take place in the thermoresistive sensing element of a fire detector as a result of the manifestation of the Joule-Lenz effect. This mathematical notation belongs to the class of equations from mathematical physics, and its solution was derived by means of the Hankel integral transformation. This description makes it possible to determine the dynamics of temperature distribution changes in a sensing element. The peculiarity of such a mathematical notation is that the parameters of the electric current flowing through the thermoresistive sensing element are not subject to restrictions. When using a mathematical description, the physical and geometrical parameters of the thermoresistive sensing element, as well as the nature of the alteration of electric current, should be taken into account.

2. The structure and parameters of the model of the thermoresistive sensing element of a fire detector in the class of transfer functions have been substantiated. It is shown that the transfer function of the inertial link could be used as such a transfer function with an error whose value does not exceed $4.6 \%$. The peculiarity of the transfer function of the thermoresistive element of a fire detector is that its transmission coefficient includes its time constant as a multiplicative component.

3. It has been shown that the ratio of reactions of the thermoresistive sensing element of a fire detector, determined in the a priori defined time moments, to the test influence in the form of an electric current pulse of a trian- 
gular shape is indifferent to the parameters of the pulse and the characteristics of the sensing element. The value of this relationship is used to formalize procedures whose execution ensures the implementation of the method for determining the time parameter - the time constant of fire detectors with a thermoresistive sensing element.

\section{References}

1. Wadoud, A. A., El Eissawi, H. M., Saleh, A. A. (2017). Protection of High Ceiling Nuclear Facilities Using Photoelectric Sensors and Infrared Fire Detectors. Arab Journal of Nuclear Science and Applications, 50 (1), 194-203. Available at: http://www.esnsa-eg.com/ download/researchFiles/(19)\%20\%20\%20\%20\%20123-15.pdf

2. Dinh, T., Phan, H.-P., Qamar, A., Woodfield, P., Nguyen, N.-T., Dao, D. V. (2017). Thermoresistive Effect for Advanced Thermal Sensors: Fundamentals, Design Considerations, and Applications. Journal of Microelectromechanical Systems, 26 (5), 966-986. doi: https://doi.org/10.1109/jmems.2017.2710354

3. Szelmanowski, A., Zieja, M., Pazur, A., Głyda, K. (2019). Studying the Dynamic Properties of Thermoelectric Fire Detectors in Terms of False Tripping of an Air Fire Suppression System. Engineer of the XXI Century, 103-120. doi: https://doi.org/10.1007/ 978-3-030-13321-4_10

4. Choi, M.-S., Lee, K.-O. (2018). Study on Influence of Air Flow of Ceiling Type Air Conditioner on Fire Detector Response. Fire Science and Engineering, 32 (5), 40-45. doi: https://doi.org/10.7731/kifse.2018.32.5.040

5. Jevtić, R., Blagojević, M. (2017). Smoke and heat detectors arrangement in hallways. Safety Engineering, 7 (2). doi: https:// doi.org/10.7562/se2017.7.02.04

6. Kalchenko, Y., Abramov, Y. (2018). Methods of heat detectors technical condition control. Problemy pozhezhnoi bezpeky, 44, 44-48. Available at: https://nuczu.edu.ua/sciencearchive/ProblemsOfFireSafety/vol44/Kalchenko.pdf

7. Lugovkin, V. V., Zhuravlev, S. Y., Bulatova, V. V. (2019). Mathematic Simulation of Thermal Sensor Operation at Various Temperature Conditions of Controlled Media. 2019 International Russian Automation Conference (RusAutoCon). doi: https:// doi.org/10.1109/rusautocon.2019.8867603

8. Lu, K. H., Mao, S. H., Wang, J., Lu, S. (2017). Numerical simulation of the ventilation effect on fire characteristics and detections in an aircraft cargo compartment. Applied Thermal Engineering, 124, 1441-1446. doi: https://doi.org/10.1016/ j.applthermaleng.2017.06.128

9. Kushnir, A., Kopchak, B., Gavryliuk, A. (2021). Operational algorithm for a heat detector used in motor vehicles. Eastern-European Journal of Enterprise Technologies, 3 (10 (111)), 6-18. doi: https://doi.org/10.15587/1729-4061.2021.231894

10. Kushnir, A., Kopchak, B., Gavryliuk, A. (2020). The Development of Operation Algorithm of Heat Detector with Variable Response Parameters. 2020 IEEE XVIth International Conference on the Perspective Technologies and Methods in MEMS Design (MEMSTECH). doi: https://doi.org/10.1109/memstech49584.2020.9109436

11. Güllüce, Y., Çelik, R. N. (2020). FireAnalyst: An effective system for detecting fire geolocation and fire behavior in forests using mathematical modeling. Turkish Journal of Agriculture and Forestry, 44 (2), 127-139. doi: https://doi.org/10.3906/tar-1907-11

12. Wang, J., Li, G., Shi, L., Xie, Q., Zhang, S. (2018). A mathematical model for heat detector activation time under ship fire in a longnarrow space. Ocean Engineering, 159, 305-314. doi: https://doi.org/10.1016/j.oceaneng.2018.04.012

13. Sharma, S., Sharma, A., Vishwakarma, P., Sharma, M. (2021). A theoretical framework representing seminal fire detection system design using shape memory polymer embedded with carbon nanotubes sponge. Materials Today: Proceedings, 44, 1617-1620. doi: https://doi.org/10.1016/j.matpr.2020.11.816

14. Yuan, L., Thomas, R. A., Rowland, J. H., Zhou, L. (2018). Early fire detection for underground diesel fuel storage areas. Process Safety and Environmental Protection, 119, 69-74. doi: https://doi.org/10.1016/j.psep.2018.07.022

15. Sharma, V., Varma, A. S., Singh, A., Singh, D., Yadav, B. P. (2018). A Critical Review on the Application and Problems Caused by False Alarms. Intelligent Communication, Control and Devices, 371-380. doi: https://doi.org/10.1007/978-981-10-5903-2_38

16. Malykhina, G. F., Guseva, A. I., Militsyn, A. V. (2017). Early fire prevention in the plant. 2017 International Conference on Industrial Engineering, Applications and Manufacturing (ICIEAM). doi: https://doi.org/10.1109/icieam.2017.8076375

17. Saeed, F., Paul, A., Karthigaikumar, P., Nayyar, A. (2019). Convolutional neural network based early fire detection. Multimedia Tools and Applications, 79 (13-14), 9083-9099. doi: https://doi.org/10.1007/s11042-019-07785-w

18. Sowah, R., Ampadu, K. O., Ofoli, A. R., Koumadi, K., Mills, G. A., Nortey, J. (2019). A Fire-Detection and Control System in Automobiles: Implementing a Design That Uses Fuzzy Logic to Anticipate and Respond. IEEE Industry Applications Magazine, 25 (2), 57-67. doi: https://doi.org/10.1109/mias.2018.2875189

19. Jang, H.-Y., Hwang, C.-H. (2020). Test Method Using Shield-cup for Evaluating Response Characteristics of Fire Detectors. Fire Science and Engineering, 34 (4), 36-44. doi: https://doi.org/10.7731/kifse.8696ecf9

20. Hong, S. H., Kim, D. S., Choi, K. O. (2017). A Study on the Classification of Domestic Fire Detector using Response Time Index. Journal of the Korean Society of Safety, 32 (2), 46-51. doi: https://doi.org/10.14346/JKOSOS.2017.32.2.46

21. Yoon, G.-Y., Han, H.-S., Mun, S.-Y., Park, C.-H., Hwang, C.-H. (2020). DB Construction of Activation Temperature and Response Time Index for Domestic Fixed-temperature Heat Detectors in Ceiling Jet Flow. Fire Science and Engineering, 34 (3), 35-42. doi: https://doi.org/10.7731/kifse.103eea8f

22. Abramov, Yu. O., Kalchenko, Ya. Yu. (2016). Teplovi pozhezhni spovishchuvachi ta yikh vyprobuvannia. Kharkiv: NUTsZU, 120. 